University of New Orleans

ScholarWorks@UNO

7-6-2007

\title{
Wide-angle, high-extinction-ratio, infrared polarizing beam splitters using frustrated total internal reflection by an embedded centrosymmetric multilayer
}

Siva R. Perla

R. M.A. Azzam

University of New Orleans, razzam@uno.edu

Follow this and additional works at: https://scholarworks.uno.edu/ee_facpubs

Part of the Electrical and Electronics Commons

\section{Recommended Citation}

Siva R. Perla and R. M. A. Azzam, "Wide-angle, high-extinction-ratio, infrared polarizing beam splitters using frustrated total internal reflection by an embedded centrosymmetric multilayer," Appl. Opt. 46, 4604-4612 (2007).

This Article is brought to you for free and open access by the Department of Electrical Engineering at ScholarWorks@UNO. It has been accepted for inclusion in Electrical Engineering Faculty Publications by an authorized administrator of ScholarWorks@UNO. For more information, please contact scholarworks@uno.edu. 


\title{
Wide-angle, high-extinction-ratio, infrared polarizing beam splitters using frustrated total internal reflection by an embedded centrosymmetric multilayer
}

\author{
Siva R. Perla and R. M. A. Azzam* \\ Department of Electrical Engineering, University of New Orleans, New Orleans, Louisiana 70148, USA \\ *Corresponding author: razzam@uno.edu
}

Received 24 January 2007; revised 13 March 2007; accepted 13 March 2007; posted 14 March 2007 (Doc. ID 79350); published 6 July 2007

\begin{abstract}
A centrosymmetric multilayer stack of two transparent thin-film materials, which is embedded in a high-index prism, is designed to function as an efficient polarizer or polarizing beam splitter (PBS) under conditions of frustrated total internal reflection over an extended range of incidence angles. The $S(\mathrm{LH})^{k} \mathrm{LHL}(\mathrm{HL})^{k} S$ multilayer structure consists of a high-index center layer $H$ sandwiched between two identical low-index films $L$ and high-index-low-index bilayers repeated ( $k$ times) on both sides of the central trilayer maintaining the symmetry of the entire stack. For a given set of refractive indices, all possible solutions for the thicknesses of the layers that suppress the reflection of $p$-polarized light at a specified angle, and the associated reflectance of the system for the orthogonal $s$ polarization, are determined. The angular and spectral sensitivities of polarizing multilayer stacks employing $3,7,11,15$, and 19 layers of $\mathrm{BaF}_{2}$ and $\mathrm{PbTe}$ thin films embedded in a $\mathrm{ZnS}$ prism, operating at $\lambda=10.6 \mu \mathrm{m}$, are presented. The 15- and 19-layer stack designs achieve extinction ratios (ER) $>30 \mathrm{~dB}$ in both reflection and transmission over a $46^{\circ}-56^{\circ}$ field of view inside the prism. Spectral analysis reveals additional discrete polarizing wavelengths other than the design wavelength $(\lambda=10.6 \mu \mathrm{m})$. The 11-, 15-, and 19-layer designs function as effective $s$-reflection polarizers $\left(\left|R_{s}\right|^{2}>99 \%\right)$ over a $2-3 \mu \mathrm{m}$ bandwidth. The effect of decreasing the refractive index contrast between the $H$ and $L$ layers on the resulting ER is also considered. (C) 2007 Optical Society of America
\end{abstract}

OCIS codes: $120.2130,260.5430,230.5440$.

\section{Introduction}

Thin-film polarizers and polarizing beam splitters (PBS) are well known [1-5] and are based on the destructive interference of light for one linear polarization ( $p$ or $s$ ) and the nearly fully constructive interference for the orthogonal polarization at oblique incidence. Considerable progress has recently been made in the design of broadband, wide-angle, polarizers and PBS [6-9]. Previous work by the authors, that dealt with the polarizing properties of centrosymmetric trilayer stacks $[10,11]$, is extended here to any odd number of layers to achieve better angular and spectral response.

The PBS, Fig. 1, uses a centrosymmetric multilayer structure $S(\mathrm{LH})^{k} \mathrm{LHL}(\mathrm{HL})^{k} S$ that consists of a highindex center layer $H$ of refractive index $n_{2}$ sand-

0003-6935/07/214604-09\$15.00/0

(C) 2007 Optical Society of America wiched between two identical low-index films $L$ of refractive index $n_{1}$ and high-index-low-index $\left(n_{2}\right.$ $-n_{1}$ ) bilayers repeated ( $k$ times) on both sides of the central trilayer maintaining the symmetry of the entire stack, which is itself embedded in a high-index prism substrate $S$ of refractive index $n_{0}$. All media are considered to be transparent, optically isotropic, and are separated by parallel-plane boundaries. We assume that $n_{0}>n_{1}, n_{2} \geq n_{0}$ and that light is incident from medium 0 at an angle $\phi_{0}$, which is greater than the critical angle $\left[\phi_{c 01}=\arcsin \left(n_{1} / n_{0}\right)\right]$ of the 01 interface, so that frustrated total internal reflection (FTIR) takes place.

The design procedure is presented in Section 2. In Section 3, all possible thickness solutions are determined for various (3, 7, 11, 15, and 19) embedded centrosymmetric multilayer stacks of given refractive indices that suppress the reflection of $p$-polarized light at each one of several angles of incidence $\phi_{0}$, 


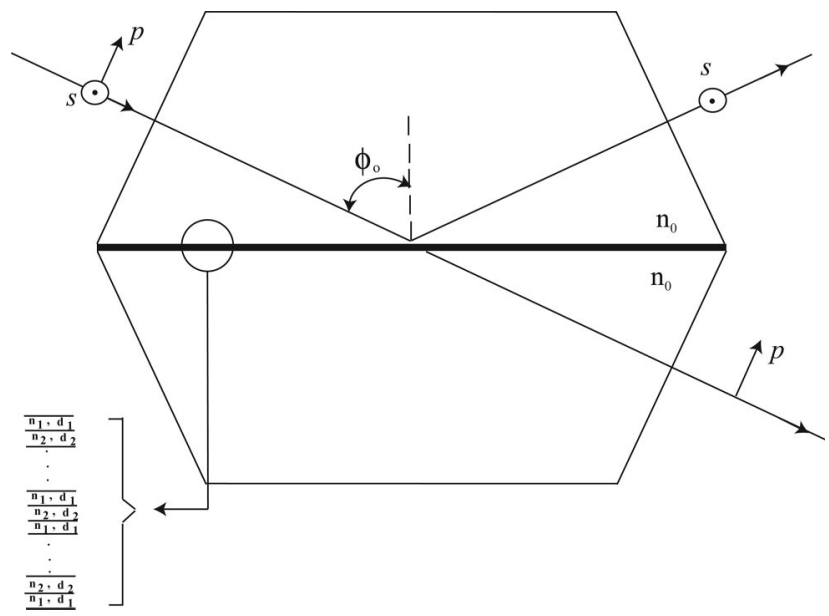

Fig. 1. Embedded centrosymmetric multilayer thin-film device as a polarizing beam splitter (PBS) operating under conditions of frustrated total internal reflection. $p$ and $s$ are the linear polarizations parallel and perpendicular to the plane of incidence, respectively, and $\phi_{0}$ is the angle of incidence.

while maintaining high reflectance for the $s$ polarization. Results are presented for IR PBS at the $\mathrm{CO}_{2}$-laser wavelength $\lambda=10.6 \mu \mathrm{m}$ that use multi- layer stacks of $\mathrm{BaF}_{2}$ and $\mathrm{PbTe}$ thin films embedded in a Cleartran [12] $(\mathrm{ZnS})$ prism.

In Section 4, the performance of various multilayer stacks that function as polarizers (or PBS) at two angles of choice (e.g., $50^{\circ}$ and $55^{\circ}$ ) is described. The 15- and 19-layer PBS designs achieve extinction ratios $(\mathrm{ER})>30 \mathrm{~dB}$ in reflection and transmission over a $46^{\circ}-56^{\circ}$ field of view inside the prism. The angular range measured in air, outside the $\mathrm{ZnS}$ prism, is twice as large, if refraction at the entrance face of the prism is accounted for using Snell's law.

In Section 5, spectral analysis of the dual-angle polarizers described in Section 4 in the range from 8 to $12 \mu \mathrm{m}$ reveals additional discrete polarizing wavelengths other than the design wavelength $(\lambda=10.6$ $\mu \mathrm{m})$. The 11-, 15-, and 19-layer designs function as effective $s$-reflection polarizers $\left(\left|R_{s}\right|^{2}>99 \%\right)$ over a 2-3 $\mu \mathrm{m}$ bandwidth. When the refractive index contrast between the $H$ and $L$ layers $\left(n_{2}-n_{1}\right)$ is reduced, the ER in transmission drops, as demonstrated in Section 6. Finally, Section 7 gives a brief summary of the paper.

\section{Design Procedure}

Consider a monochromatic light beam traveling in an ambient medium (solid prism) of refractive index $n_{0}$ and incident on an embedded centrosymmetric mul-
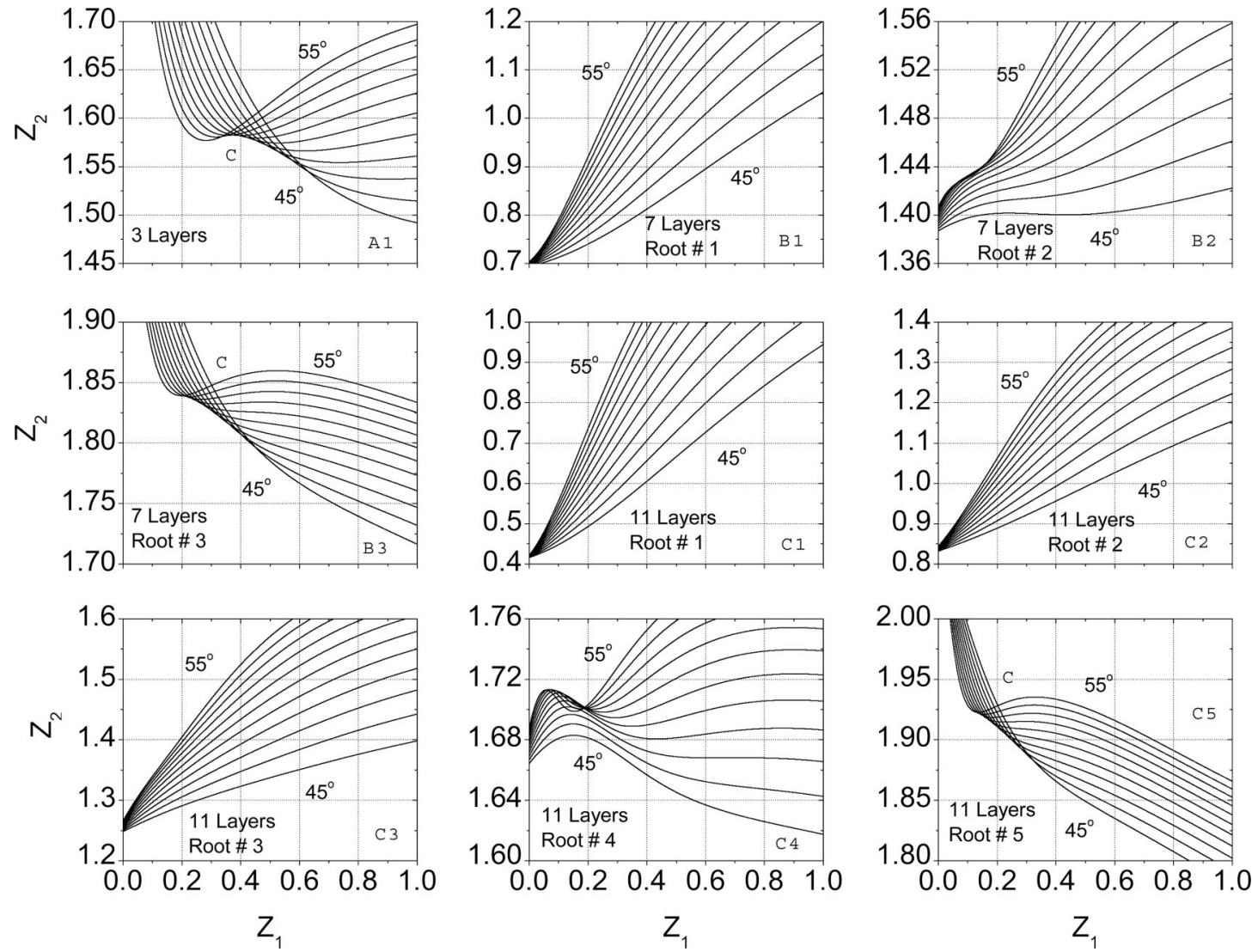

Fig. 2. $Z_{2}$ versus $Z_{1}$ such that $R_{p}=0$ at angles of incidence $\phi_{0}$ from $45^{\circ}$ to $55^{\circ}$ in steps of $1^{\circ}$ for centro-symmetric multilayer stacks with $\mathrm{BaF}_{2}$ and $\mathrm{PbTe}$ thin films embedded in a $\mathrm{ZnS}$ substrate with refractive indices $n_{0}=2.1919(\mathrm{ZnS}), n_{1}=1.3926\left(\mathrm{BaF}_{2}\right)$, and $n_{2}=$ $5.6314(\mathrm{PbTe})$ in the IR at $\lambda=10.6 \mu \mathrm{m}$. Panel A1 corresponds to a trilayer design; panels B1, B2 and B3 correspond to the seven-layer design; while panels $\mathrm{C} 1, \mathrm{C} 2, \mathrm{C} 3, \mathrm{C} 4$ and $\mathrm{C} 5$ correspond to the 11-layer design. 
tilayer structure at an angle of incidence $\phi_{0}$ with respect to the normal of the interfaces, as shown in Fig. 1. The complex-amplitude transmission and reflection coefficients $T_{v}$ and $R_{v}(v=p, s)$ of the multilayer stack for the $p$ and $s$ polarizations at oblique incidence are determined by the scattering matrix method [13]. A general expression for the complexamplitude reflection coefficient $\left(\boldsymbol{R}_{v}\right)$ for an $m$-layer centrosymmetric multilayer stack embedded in a high-index prism, as shown in Fig. 1, can be expressed as

$$
\begin{aligned}
R_{v} & =\frac{a_{n} X_{2}^{n}+a_{n-1} X_{2}^{n-1}+\cdots+a_{0}}{b_{n} X_{2}^{n}+b_{n-1} X_{2}^{n-1}+\cdots+b_{0}}, \\
v & =p, s .
\end{aligned}
$$

In Eq. (1), $n=(m-1) / 2$, and $a_{n}, a_{n-1}, \ldots, a_{0} ; b_{n}$, $b_{n-1}, \ldots, b_{0}$ are functions of the Fresnel interface reflection coefficients $r_{01 v}, r_{12 v}$, and $X_{1}$. The complex exponential functions of film thickness $X_{1}$ and $X_{2}$ are given by

$$
X_{i}=\exp \left(-j \pi Z_{i} \cos \phi_{\mathrm{i}}\right),
$$

where $Z_{i}$ is the thickness of the $i$ th film normalized to the quarter-wave thickness at normal incidence, i.e.,

$$
Z_{i}=\frac{4 d_{i} n_{i}}{\lambda} .
$$

In Eq. (2), $\phi_{i}$ is the angle of refraction in the $i$ th layer, and in Eq. (3) $n_{i}, d_{i}$ are the refractive index and metric thickness of the $i$ th layer, respectively, and $\lambda$ is the vacuum wavelength of light.

The Fresnel complex-amplitude reflection coefficients of the $i j$ interface for the $p$ and $s$ polarizations are given by [13]:

$r_{i j p}=\frac{n_{j} \cos \phi_{i}-n_{i} \cos \phi_{j}}{n_{j} \cos \phi_{i}+n_{i} \cos \phi_{j}}, \quad r_{i j s}=\frac{n_{i} \cos \phi_{i}-n_{j} \cos \phi_{j}}{n_{i} \cos \phi_{i}+n_{j} \cos \phi_{j}}$.

To suppress the $v$ polarization on reflection, we set $R_{v}=0$ in Eq. (1), which gives

$$
a_{n} X_{2}^{n}+a_{n-1} X_{2}^{n-1}+\cdots+a_{0}=0 \text {. }
$$

Depending on the order $n$ of the polynomial in Eq. (5), multiple roots for $X_{2}$ are obtained for designs with number of layers $m>3$, corresponding to multiple solutions sets $\left(X_{1}, X_{2}\right)$ that suppress the $v$ polarization.

When the refractive indices and angle of incidence are such that $n_{1} / n_{0}<\sin \phi_{0}$, FTIR takes place at the 01 interface at $\phi_{0}$ and the light field becomes evanescent in medium 1 (the low-index film). In this case, $\cos \phi_{1}$ is pure-imaginary, and $X_{1}$ is real in the range of $0 \leq X_{1} \leq 1$. Because we also choose $n_{2} \geq n_{0}$, the angle of refraction $\phi_{2}$ in the high-index layers is real,
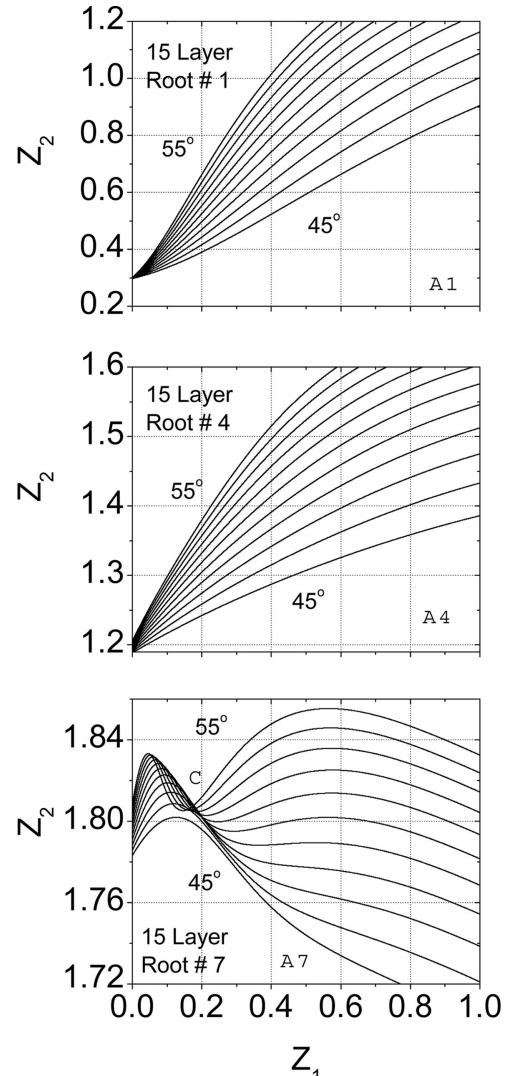
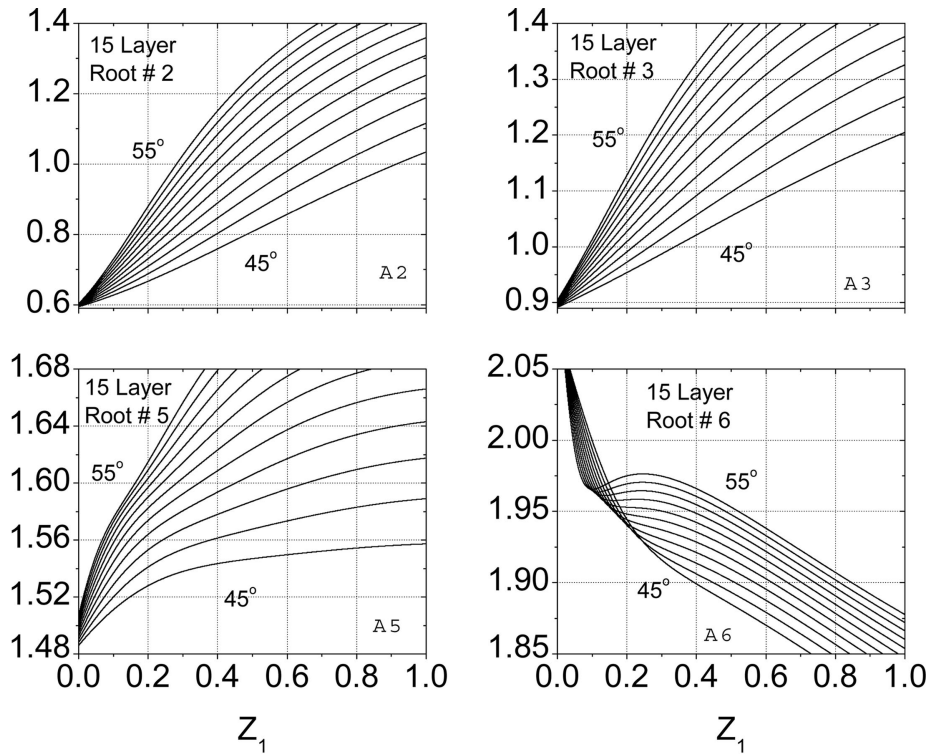

Fig. 3. $Z_{2}$ versus $Z_{1}$ such that $R_{p}=0$ at angles of incidence $\phi_{0}$ from $45^{\circ}$ to $55^{\circ}$ in steps of $1^{\circ}$ for the same material system as described in the caption of Fig. 2 for a 15-layer centrosymmetric design. 

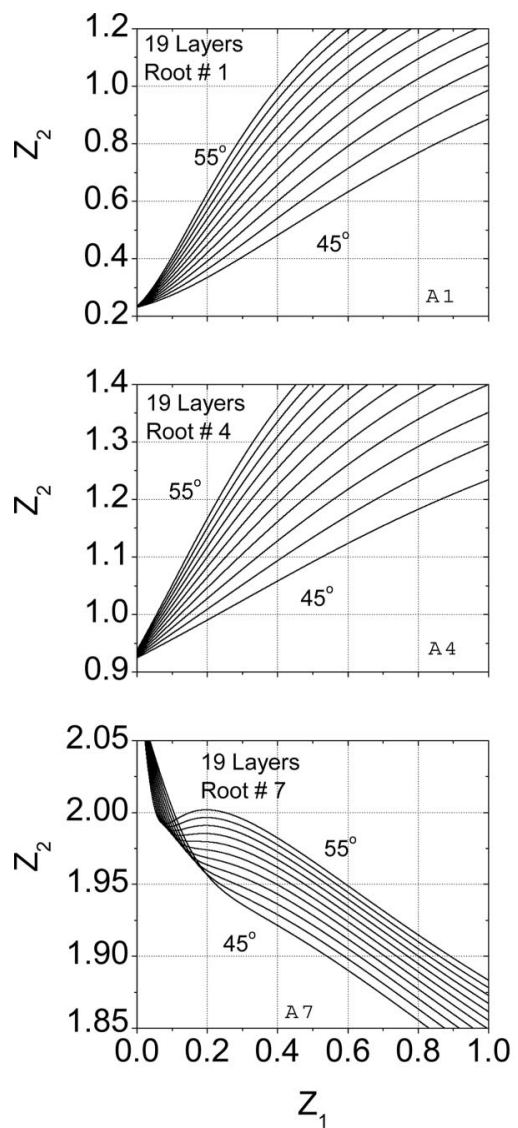
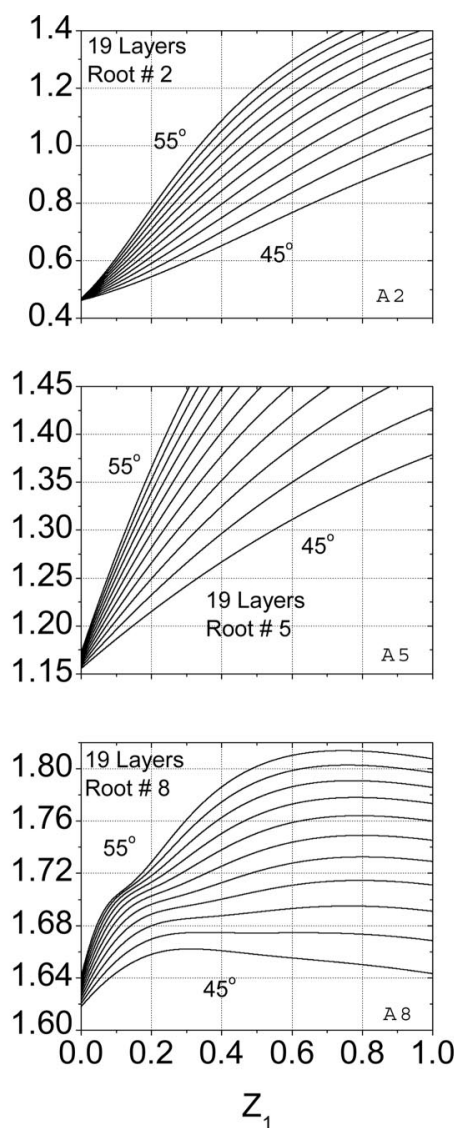
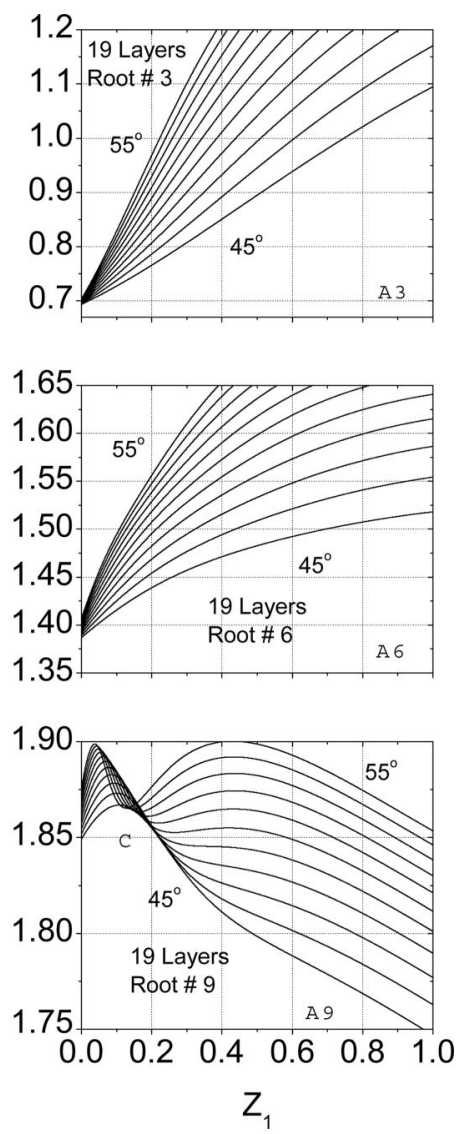

Fig. 4. $Z_{2}$ versus $Z_{1}$ such that $R_{p}=0$ at angles of incidence $\phi_{0}$ from $45^{\circ}$ to $55^{\circ}$ in steps of $1^{\circ}$ for the same material system as described in the caption of Fig. 2 for a 19-layer centrosymmetric design.

which makes $X_{2}$ a pure phase factor, so that $\left|X_{2}\right|$ $=1$. For each real value of $X_{1}$ in the range $0 \leq X_{1}$ $\leq 1$, we find that $\left|X_{2}\right|=1$ for each root of Eq. (5). Therefore, there are an infinite number of solution sets $\left(X_{1}, X_{2}\right)$ that satisfy Eq. (5), so that $R_{v}=0$. The corresponding solution sets of normalized film thicknesses $\left(Z_{1}, Z_{2}\right)$ are determined subsequently using Eq. (2).

An acceptable design must have a high reflectance $R_{v}=\left|R_{v}\right|^{2}$ for the unsuppressed orthogonal polarization $v$. In general, this reflectance increases as the normalized thickness $Z_{1}$ (of the low-index layers that support the evanescent field) and the angle of incidence $\phi_{0}$ are increased.

Table 1. Normalized $\left(z_{1}, z_{2}\right)$ and Metric $\left(d_{1}, d_{2}\right)$ Thicknesses Corresponding to the Point of Intersection of the $50^{\circ}$ and $55^{\circ}$ $Z_{2}$-versus- $Z_{1}$ Curves in Panel A1 of Fig. 2, Panel B3 of Fig. 2, Panel C5 of Fig. 2, Panel A7 of Fig. 3, and Panel A9 of Fig. 4 for the 3-, 7-, 11-, 15-, and 19-Layer Designs, Respectively

\begin{tabular}{ccccc}
\hline $\begin{array}{c}\text { Number of } \\
\text { Layers }\end{array}$ & $Z_{1}$ & $Z_{2}$ & $\begin{array}{c}d_{1} \\
(\mu \mathrm{m})\end{array}$ & $\begin{array}{c}d_{2} \\
(\mu \mathrm{m})\end{array}$ \\
\hline 3 & 0.377275 & 1.588134 & 0.71792 & 0.74734 \\
7 & 0.234571 & 1.840903 & 0.44637 & 0.86628 \\
11 & 0.188795 & 1.701440 & 0.35926 & 0.80066 \\
15 & 0.173570 & 1.807341 & 0.33029 & 0.85049 \\
19 & 0.149774 & 1.866958 & 0.28501 & 0.87855 \\
\hline
\end{tabular}
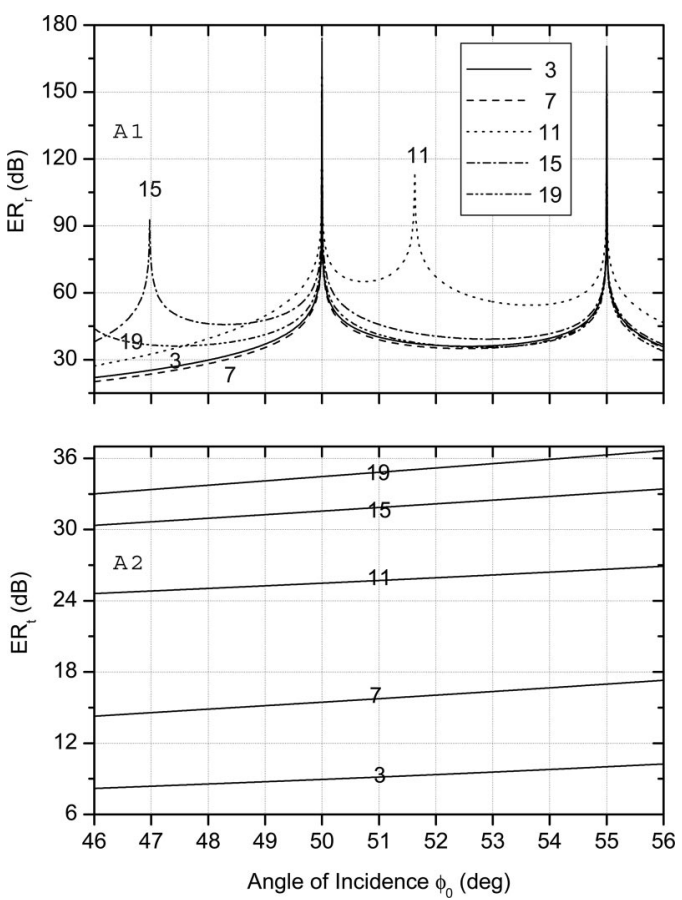

Fig. 5. Extinction ratios in reflection and transmission $\left(\mathrm{ER}_{r}\right.$ and $\mathrm{ER}_{t}$ ) in decibels for various centrosymmetric multilayer stacks with 3-19 layers embedded in a $\mathrm{ZnS}$ substrate are plotted versus the angle of incidence $\phi_{0}$ from $46^{\circ}$ to $56^{\circ}$. The material system is the same as given in the caption of Fig. 2, and the metric film thicknesses associated with different multilayer stacks are listed in Table 1. 
Table 2. Discrete Angles at Which the ER $r$ Reaches a Peak in Panel A1 of Fig. 5

\begin{tabular}{clrr}
\hline $\begin{array}{c}\text { Number of } \\
\text { Layers }\end{array}$ & $\begin{array}{c}\phi_{0} \\
(\mathrm{deg})\end{array}$ & $\begin{array}{c}\mathrm{ER}_{r} \\
(\mathrm{~dB})\end{array}$ & $\begin{array}{r}\mathrm{ER}_{t} \\
(\mathrm{~dB})\end{array}$ \\
\hline 3 & $50^{\circ}$ & 162 & 8.9 \\
& $55^{\circ}$ & 168 & 10.0 \\
7 & $50^{\circ}$ & 168 & 15.4 \\
& $55^{\circ}$ & 168 & 16.9 \\
11 & $50^{\circ}$ & 180 & 25.5 \\
& $51.63^{\circ}$ & 114 & 25.8 \\
& $55^{\circ}$ & 170 & 26.6 \\
& $46.97^{\circ}$ & 92 & 30.6 \\
& $50^{\circ}$ & 173 & 31.5 \\
& $55^{\circ}$ & 171 & 33.1 \\
& $50^{\circ}$ & 161 & 34.5 \\
& $55^{\circ}$ & 159 & 36.2 \\
\hline
\end{tabular}

\section{3. $\mathrm{BaF}_{2}-\mathrm{PbTe}$ Multilayer Polarizers Operating at $\lambda=10.6 \mu \mathrm{m}$}

Figures 2-4 show $Z_{2}$ versus $Z_{1}$ such that $R_{p}=0$ at angles of incidence $\phi_{0}$ from $45^{\circ}$ to $55^{\circ}$ in steps of $1^{\circ}$ for various centrosymmetric multilayer stacks with 3,7 , 11, 15, and 19 layers of $\mathrm{BaF}_{2}$ and $\mathrm{PbTe}$ thin films embedded in a ZnS substrate with refractive indices $n_{0}=2.1919(\mathrm{ZnS}), n_{1}=1.3926\left(\mathrm{BaF}_{2}\right)$, and $n_{2}=$ $5.6314(\mathrm{PbTe})$ in the IR. (The refractive indices of
$\mathrm{ZnS}, \mathrm{BaF}_{2}$, and $\mathrm{PbTe}$ are calculated to four decimal places using published dispersion relations [14].) In Fig. 2, panel A1 corresponds to the trilayer design with one root; panels B1, B2, and B3 correspond to the seven-layer design with three roots; while panels $\mathrm{C} 1, \mathrm{C} 2, \mathrm{C} 3, \mathrm{C} 4$, and $\mathrm{C} 5$ correspond to the 11-layer design with five roots. Figure 3 shows the corresponding results for the 15-layer design with seven roots, while Fig. 4 shows the results for the 19-layer design with nine roots. Every point $\left(Z_{1}, Z_{2}\right)$ on each curve in Figs. 2-4 represents a thickness solution that achieves a polarizer $\left(R_{p}=0\right)$ at the angle of incidence marked by that curve. For designs with 3-19 layers, the $Z_{2}$-versus- $Z_{1}$ curves that correspond to some roots are clustered and mutually intersecting in a small region of the $Z_{2}$-versus- $Z_{1}$ plane. By selecting an intersection point $\left(Z_{1}, Z_{2}\right)$ of two curves in this cluster region we obtain an $R_{p}=0$ polarizer at two angles of incidence simultaneously. For the results presented in Sections 4 and 5 we choose, for each design with $3-19$ layers, the intersection point of the $50^{\circ}$ and $55^{\circ}$ curves that correspond to the root with the highest root number (panel A1 of Fig. 2, panel B3 of Fig. 2, panel C5 of Fig. 2, panel A7 of Fig. 3, and panel A9 of Fig. 4 for the 3-, 7-, 11-, 15-, and 19-layer designs, respectively). The point of intersection of the $50^{\circ}$ and $55^{\circ}$ curves for the root with the highest root number yields a design with $R_{p} \approx 0$ and high reflectance $\left|R_{s}\right|^{2}$
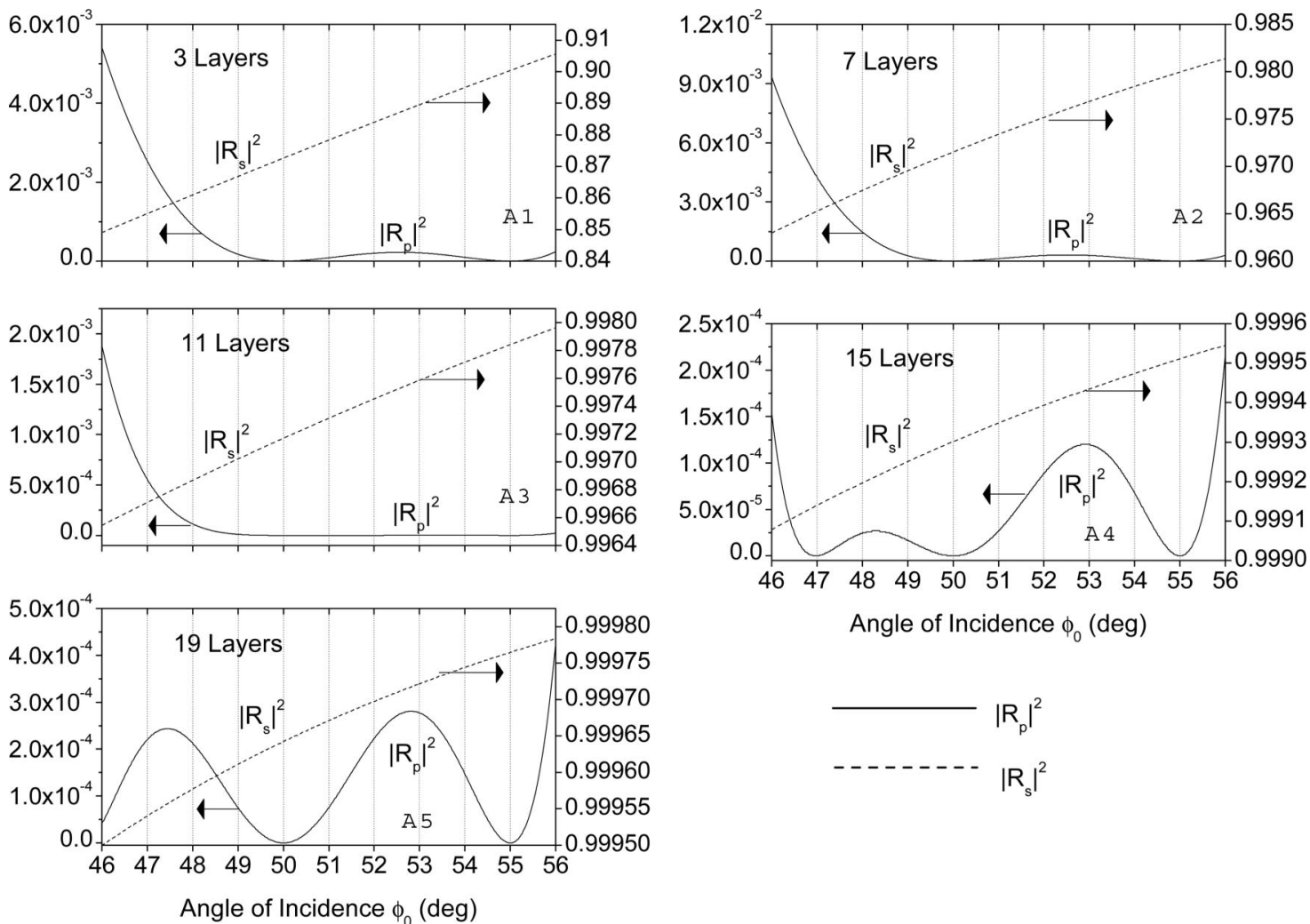

Angle of Incidence $\phi_{0}(\mathrm{deg})$

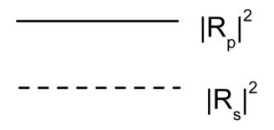

Fig. 6. Reflectances $\left|R_{p}\right|^{2}$ and $\left|R_{s}\right|^{2}$ for various centro-symmetric multilayer stacks with 3-19 layers embedded in a ZnS substrate are plotted versus the angle of incidence $\phi_{0}$ from $46^{\circ}$ to $56^{\circ}$. The material system is the same as given in the caption of Fig. 2 , and the metric film thicknesses associated with different multilayer designs are listed in Table 1. 
for all angles of incidence from $45^{\circ}$ to $55^{\circ}$. Table 1 lists the normalized and metric film thicknesses $\left(Z_{1}, Z_{2}\right)$ and $\left(d_{1}, d_{2}\right)$ obtained by the above procedure for the 3-, 7-, 11-, 15-, and 19-layer designs.

\section{Angular Sensitivity}

This specific comparison between different multilayer designs is based on the thickness solutions $\left(Z_{1}, Z_{2}\right) \leftrightarrow\left(d_{1}, d_{2}\right)$ listed in Table 1 . For the angle sensitivity, the metric film thicknesses $\left(d_{1}, d_{2}\right)$ and wavelength $\lambda=10.6 \mu \mathrm{m}$ for each multilayer design are kept constant and the angle of incidence $\phi_{0}$ is varied from $46^{\circ}$ to $56^{\circ}$. In panels A1 and A2 of Fig. 5, we compare the extinction ratios in reflection and transmission $\left(\mathrm{ER}_{r}\right.$ and $\mathrm{ER}_{t}$ in decibels), respectively, versus angle of incidence $\phi_{0}$, for the 3-, 7-, 11-, 15-, and 19-layer designs. The 15- and 19-layer designs achieve $\mathrm{ER}_{r}$ and $\mathrm{ER}_{t}>30 \mathrm{~dB}$ over a $10^{\circ}$ internal field of view (from $46^{\circ}$ to $56^{\circ}$ ). If one accounts for the refraction of light from air to the high-index $\mathrm{ZnS}$ substrate, the angular bandwidth in air is more than double that which is indicated in Fig. 5. The extinction ratio $\mathrm{ER}_{r}$ is $>150 \mathrm{~dB}$ at $50^{\circ}$ and $55^{\circ}$ for all designs because each thickness solution set corresponds to $\left(Z_{1}, Z_{2}\right)$ at the point of intersection of the $50^{\circ}$ and $55^{\circ}$ curves. Table 2 lists the discrete angles at which $\mathrm{ER}_{r}$ in panel A1 of Fig. 5 reaches a peak.

Panels A1, A2, A3, A4, and A5 of Fig. 6 show the intensity reflectances $\left|R_{p}\right|^{2}$ and $\left|R_{s}\right|^{2}$ as functions of the angle of incidence $\phi_{0}$ from $46^{\circ}$ to $56^{\circ}$ for 3-, 7-, 11-, 15-, and 19-layer designs, respectively. (All parameters are the same as those used in Fig. 5.) As the number of layers increase, $\left|R_{s}\right|^{2}$ increases from $84 \%$ to $99.98 \%$, while $\left|R_{p}\right|^{2}$ remains $<0.01$ over a $10^{\circ}$ internal field of view $\left(46^{\circ}\right.$ to $\left.56^{\circ}\right)$. The 15-layer design achieves $\left|R_{p}\right|^{2}<0.00025$ and $\left|R_{s}\right|^{2}>0.999$, while the 19-layer design achieves $\left|R_{p}\right|^{2}<0.0005$ and $\left|R_{s}\right|^{2}>0.9995$ over a $10^{\circ}$ field of view $\left(46^{\circ}\right.$ to $\left.56^{\circ}\right)$. Hence good PBS with high extinction ratios $(>30$ $\mathrm{dB}$ ) in both reflection and transmission are achieved over a good range of incidence angles.

\section{Spectral Sensitivity}

The spectral sensitivities of the multilayer designs obtained in Section 4 are now analyzed. For the spectral sensitivity, the metric film thicknesses $\left(d_{1}, d_{2}\right)$ and the angle of incidence $\phi_{0}=50^{\circ}$ for each multilayer design are kept constant, and the wavelength $\lambda$ is scanned from 8 to $12 \mu \mathrm{m}$. The dispersion of all the
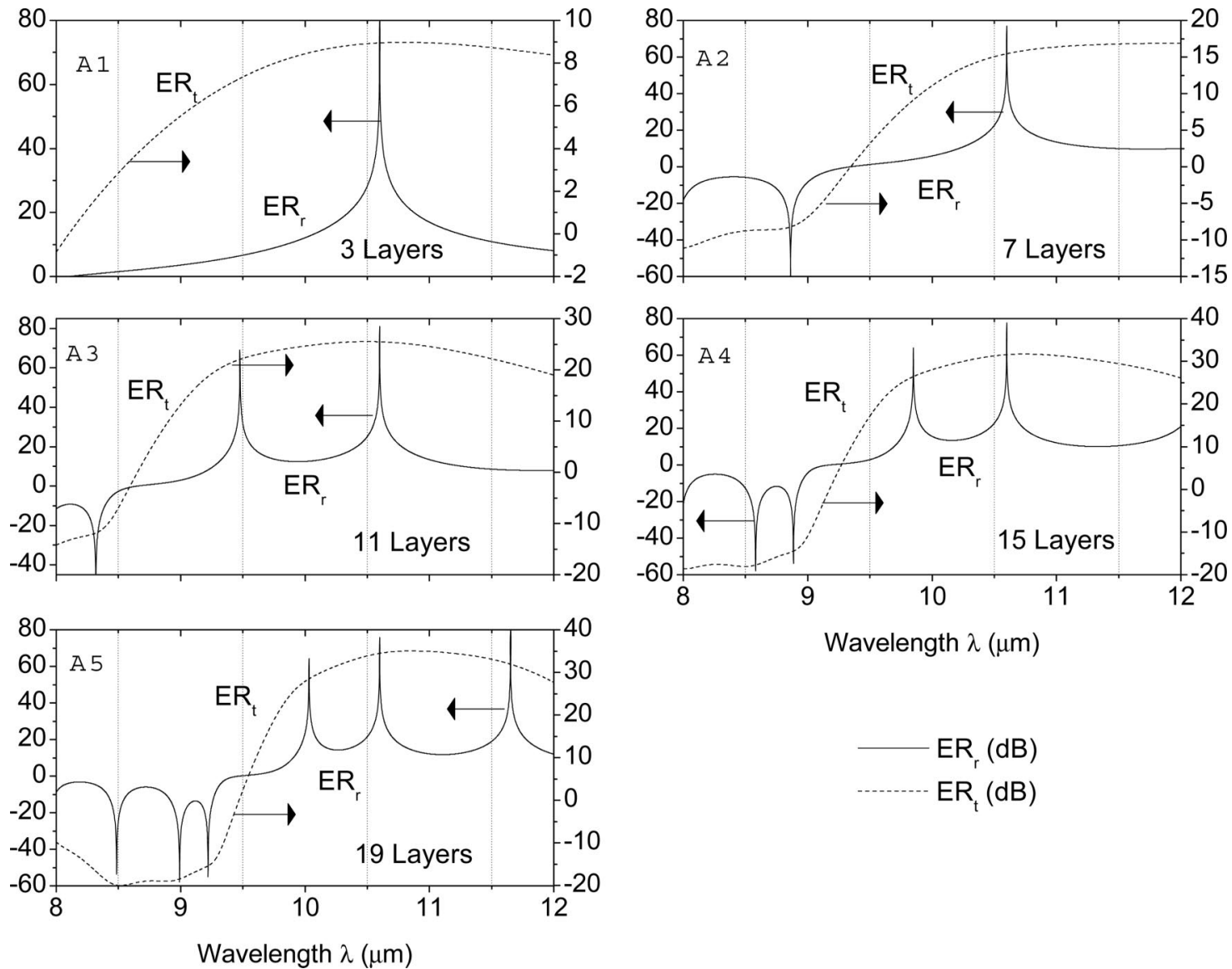

Wavelength $\lambda(\mu \mathrm{m})$



Fig. 7. Extinction ratios in reflection and transmission $\left(\mathrm{ER}_{r}\right.$ and $\left.\mathrm{ER}_{t}\right)$ in decibels for various centrosymmetric multilayer stacks with $3-19$ layers embedded in a $\mathrm{ZnS}$ substrate are plotted versus wavelength $8 \leq \lambda \leq 12 \mu \mathrm{m}$. The material system is the same as given in the caption of Fig. 2, and the metric film thicknesses associated with different multilayer stack designs are listed in Table 1. 
Table 3. Discrete Wavelengths at Which the $\mathrm{ER}_{r}$ of Fig. 7 Reaches a Positive or Negative Peak

\begin{tabular}{cccr}
\hline & \multicolumn{3}{c}{$\phi_{0}=50^{\circ}$} \\
\cline { 2 - 4 } $\begin{array}{c}\text { Number of } \\
\text { Layers }\end{array}$ & $\lambda$ & $\begin{array}{c}\mathrm{ER}_{r} \\
(\mu \mathrm{m})\end{array}$ & $\begin{array}{r}\mathrm{ER}_{t} \\
(\mathrm{~dB})\end{array}$ \\
\hline 3 & 10.6 & 82.43 & 8.95 \\
7 & 8.862 & -66.08 & -8.21 \\
& 10.6 & 77.05 & 15.44 \\
11 & 8.318 & -60.53 & -11.87 \\
& 9.477 & 69.06 & 22.05 \\
& 10.6 & 81.08 & 25.48 \\
15 & 8.582 & -57.91 & -17.72 \\
& 8.886 & -53.9 & -14.48 \\
& 9.85 & 63.92 & 26.44 \\
19 & 10.6 & 77.62 & 31.55 \\
& 8.486 & -53.73 & -19.94 \\
& 8.991 & -57.91 & -18.51 \\
& 9.22 & -55.02 & -15.44 \\
& 10.032 & 64.28 & 28.48 \\
& 10.6 & 75.73 & 34.46 \\
& 11.652 & 80.66 & 31.92 \\
\hline
\end{tabular}

materials in this spectral region is accounted for [14]. Panels A1, A2, A3, A4, and A5 of Fig. 7 show the ER in reflection and transmission as a function of the wavelength $\lambda$ for the 3-, 7-, 11-, 15-, and 19-layer designs, respectively. The left and right $Y$ axes of each panel of Fig. 7 represent $\mathrm{ER}_{r}$ and $\mathrm{ER}_{t}$, respectively. All parameters for each panel in Fig. 7 are the same as those in Fig. 6. All the designs with 3-19 layers have a peak in $\mathrm{ER}_{r}$ at $\lambda=10.6 \mu \mathrm{m}$, which is the design wavelength. These devices also function as $R_{p}=0$ polarizers, at additional discrete wavelengths other than the design wavelength. An interesting feature of these plots in Fig. 7 is that designs with number of layers $>3$ function as orthogonal polarizers $\left(R_{s}=0\right)$ or PBS at other discrete wavelengths in the spectral range $8 \leq \lambda \leq 12 \mu \mathrm{m}$. Table 3 lists all the discrete wavelengths at which $\mathrm{ER}_{r}$ in Fig. 7 reaches a positive or negative peak. (The difference in the peak values of $\mathrm{ER}_{r}$ in decibels at $\phi_{0}=50^{\circ}$ and $\lambda=$ $10.6 \mu \mathrm{m}$ in Tables 2 and 3 is an insignificant numerical precision artifact.)

Panels A1, A2, A3, A4, and A5 of Fig. 8 show the intensity reflectances $\left|R_{p}\right|^{2}$ and $\left|R_{s}\right|^{2}$ as functions of wavelength $\lambda$ from 8 to $12 \mu \mathrm{m}$ for 3-, 7-, 11-, 15-, and 19-layer designs, respectively. All parameters for each panel in Fig. 8 are the same as those in Fig. 7. The 11-, 15-, and 19-layer designs function as effective $s$-reflection polarizers with $s$ reflectance $\left|R_{s}\right|^{2}$ $>99 \%$ over a $2-3 \mu \mathrm{m}$ spectral bandwidth.
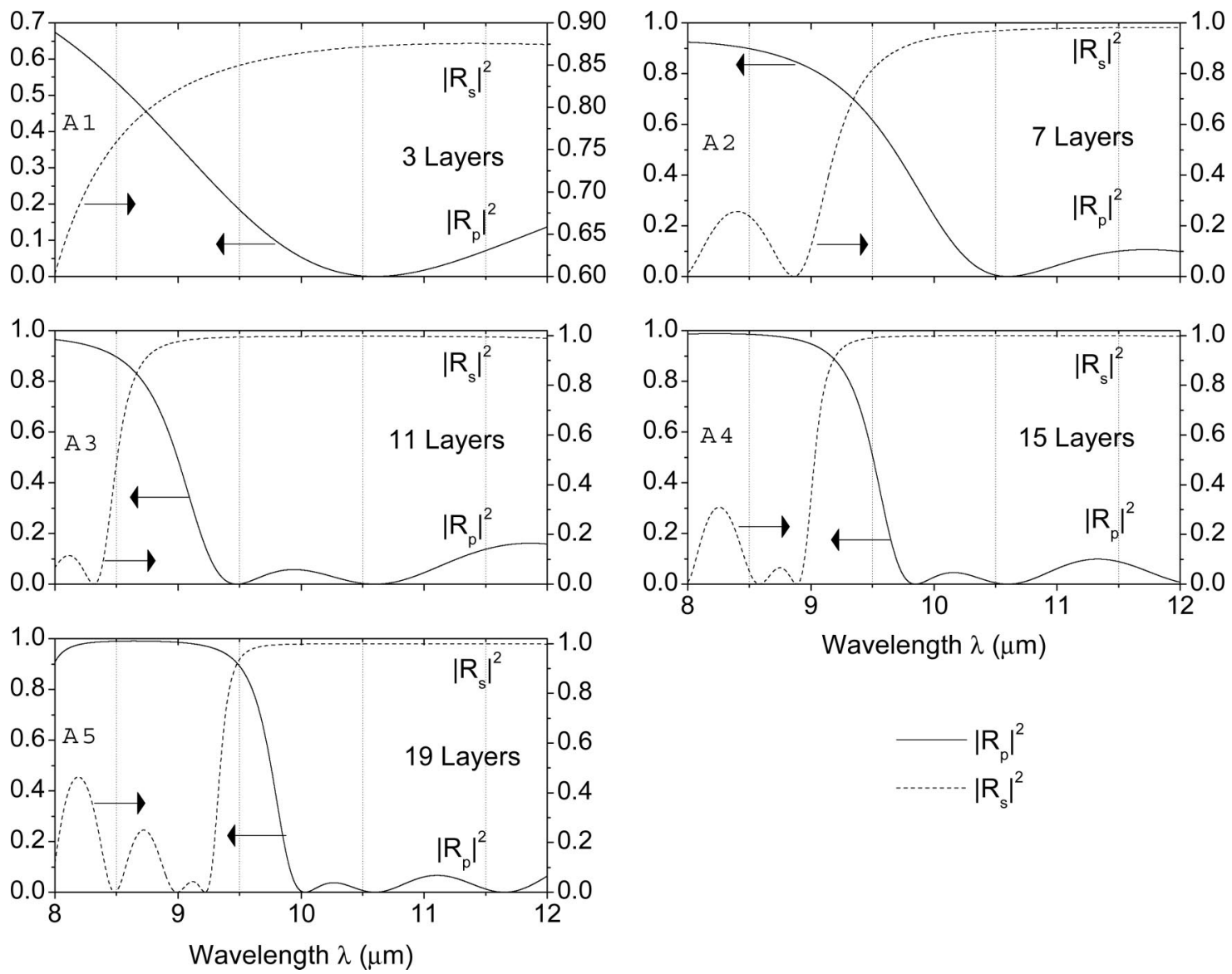

Wavelength $\lambda(\mu \mathrm{m})$

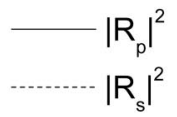

Fig. 8. Reflectances $\left|R_{p}\right|^{2}$ and $\left|R_{s}\right|^{2}$ for various centrosymmetric multilayer stacks with 3-19 layers embedded in a ZnS substrate are plotted versus wavelength $8 \leq \lambda \leq 12 \mu \mathrm{m}$. The material system is the same as given in the caption of Fig. 2 , and the metric film thicknesses associated with different multilayer stack designs are listed in Table 1. 
Table 4. Normalized $\left(Z_{1}, Z_{2}\right)$ and Metric $\left(d_{1}, d_{2}\right)$ Thicknesses for Dual-Angle $\left(50^{\circ}\right.$ and $\left.55^{\circ}\right)$ PBS Using a 15-Layer Centrosymmetric Structure with $\mathrm{BaF}_{2}\left(n_{1}=1.3926\right)$ as the Low-Index Films and $\mathrm{PbTe}, \mathrm{Ge}$ or Si ( $n_{2}=5.6314,4.0038$, and 3.4177, Respectively) as the High-Index Layers $^{a}$

\begin{tabular}{lcccc}
\hline \multicolumn{1}{c}{$n_{2}$} & \multicolumn{2}{c}{} & $\begin{array}{c}d_{1} \\
(\mu \mathrm{m})\end{array}$ & $\begin{array}{c}d_{2} \\
(\mu \mathrm{m})\end{array}$ \\
\hline $5.6314(\mathrm{PbTe})$ & 0.173570 & 1.807341 & 0.33029 & 0.85049 \\
$4.0038(\mathrm{Ge})$ & 0.122227 & 1.814895 & 0.23259 & 1.20123 \\
$3.4177(\mathrm{Si})$ & 0.083915 & 1.847262 & 0.15968 & 1.43232 \\
\hline
\end{tabular}

${ }^{a}$ The multilayer is embedded in a $\mathrm{ZnS}$ substrate with refractive index $n_{0}=2.1919$. All refractive indices correspond to the design wavelength $\lambda=10.6 \mu \mathrm{m}$.

\section{Effect of Decreasing the Refractive Index Contrast between the $\boldsymbol{H}$ and $\boldsymbol{L}$ Layers}

To investigate the effect of reducing the refractive index contrast between the high- and low-index layers of the centrosymmetric structure, calculations for the 15-layer design were repeated with $\mathrm{PbTe}\left(n_{2}\right.$ $=5.6314)$ replaced by $\mathrm{Ge}\left(n_{2}=4.0038\right)$ and $\mathrm{Si}\left(n_{2}\right.$
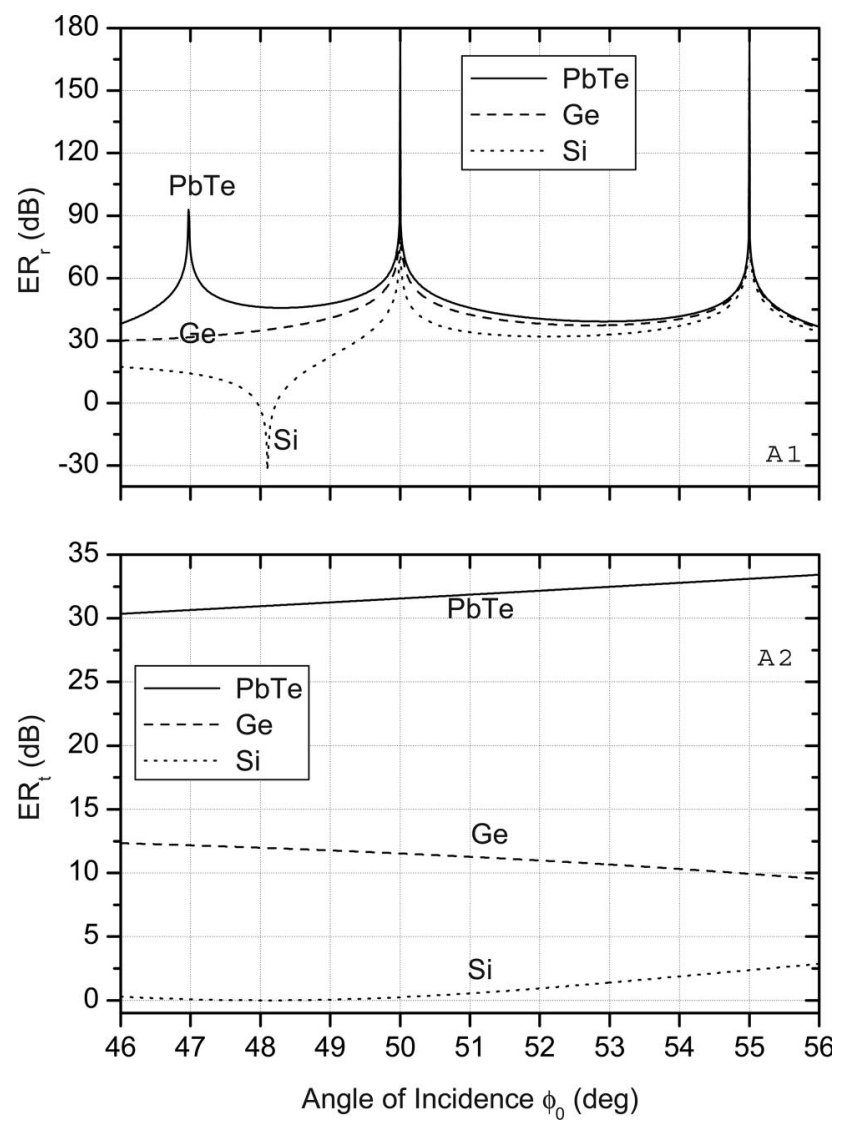

Fig. 9. Extinction ratios in reflection $\mathrm{ER}_{r}$ (panel A1) and transmission $\mathrm{ER}_{t}$ (panel A2) in decibels for a 15-layer centrosymmetric structure that uses $\mathrm{BaF}_{2}\left(n_{1}=1.3926\right)$ as the low-index films and $\mathrm{PbTe}, \mathrm{Ge}$, or $\mathrm{Si}$ ( $n_{2}=5.6314,4.0038$, and 3.4177, respectively) as the high-index layers. The multilayer is embedded in a $\mathrm{ZnS}$ substrate with refractive index $n_{0}=2.1919$. All refractive indices correspond to the operating wavelength $\lambda=10.6 \mu \mathrm{m}$. The normalized and metric film thicknesses for these 15-layer designs are listed in Table 4.
$=3.4117)$ as the high-index layers, while the $\mathrm{ZnS}$ substrate and $\mathrm{BaF}_{2}$ films remain unchanged. (The refractive indices of all materials are determined from dispersion relations given in Ref. 14.) The design corresponds to the point of intersection of the $Z_{2}$-versus- $Z_{1}$ curves at the same two angles of incidence $\phi_{0}=50^{\circ}$ and $55^{\circ}$. The normalized and metric thicknesses of the low- and high-index layers at the design point are listed in Table 4 for the three different choices of the high refractive index $n_{2}$.

The extinction ratios $\mathrm{ER}_{r}$ and $\mathrm{ER}_{t}$ in decibels for the 15-layer design at $\lambda=10.6 \mu \mathrm{m}$ are plotted versus $\phi_{0}$ in panels A1 and A2 of Fig. 9, respectively. It is apparent from Fig. 9 that the highest ER in reflection and transmission $(>30 \mathrm{~dB})$ are obtained with $\mathrm{PbTe}$ as the high-index film material. The drop in $\mathrm{ER}_{t}$ (Fig. 9, panel A2) as $\mathrm{PbTe}$ is replaced by $\mathrm{Ge}$ and $\mathrm{Si}$ is substantial and renders the performance of the device as a PBS unacceptable.

\section{Summary}

Polarizers and PBS that employ centrosymmetric multilayer stacks embedded in a high-index prism have been examined by using analytical and numerical calculations. All possible solutions (infinite in number) that suppress the $p$ or $s$ polarization in reflection are determined. (Results are presented here only for the $R_{p}=0$ polarizers.) Conditions for good operation of these devices over an extended range of incidence angles have been demonstrated.

The 15- and 19-layer designs achieve $\mathrm{ER}_{r}$ and $\mathrm{ER}_{t}>30 \mathrm{~dB}$ over a $10^{\circ}$ internal field of view $\left(46^{\circ}-\right.$ $56^{\circ}$ ). The spectral analysis reveals additional discrete wavelengths other than the design wavelength $(\lambda$ $=10.6 \mu \mathrm{m})$ at which these devices act as PBSs. The 11-, 15-, and 19-layer designs function as effective $s$-reflection polarizers with an $s$ reflectance $\left|R_{s}\right|^{2}$ $>99 \%$ over a significant $(\approx 2-3 \mu \mathrm{m})$ spectral bandwidth.

\section{References}

1. P. B. Clapham, M. J. Downs, and R. J. King, "Some applications of thin films to polarization devices," Appl. Opt. 8, 19651974 (1969).

2. D. Lees and P. Baumeister, "Versatile frustrated-totalreflection polarizer for the infrared," Opt. Lett. 4, 66-67 (1979).

3. H. A. Macleod, Thin Film Optical Filters, 2nd ed. (McGrawHill, 1986).

4. R. M. A. Azzam, "Polarizing beam splitters for infrared and millimeter waves using single-layer-coated dielectric slab or unbacked films," Appl. Opt. 25, 4225-4227 (1986).

5. B. V. Blanckenhagen, "Practical layer design for polarizing beam-splitter cubes," Appl. Opt. 45, 1539-1543 (2006).

6. J. Mouchart, J. Begel, and E. Duda, "Modified MacNeille cube polarizer for a wide angular field," Appl. Opt. 28, 2847-2853 (1989).

7. L. Li and J. A. Dobrowolski, "Visible broadband, wide-angle, thin-film multilayer polarizing beam splitter," Appl. Opt. 35, 2221-2225 (1996).

8. L. Li and J. A. Dobrowolski, "High-performance thin film polarizing beam splitter operating at angles greater than the critical angle," Appl. Opt. 39, 2754-2771 (2000). 
9. L. Li, "The design of optical thin film coatings," Opt. Photon. News 14, 24-30 (2003).

10. R. M. A. Azzam and S. R. Perla, "Polarizing properties of embedded symmetric trilayer stacks under conditions of frustrated total internal reflection," Appl. Opt. 45, 1650-1656 (2006).

11. R. M. A. Azzam and S. R. Perla "Polarizing properties of embedded symmetric trilayer stacks under conditions of frustrated total internal reflection: erratum," Appl. Opt. 46, 431-433 (2007).

12. CVD, Inc., 35 Industrial Parkway, Woburn, Mass. 01801.

13. R. M. A. Azzam and N. M. Bashara, Ellipsometry and Polarized Light (North-Holland, 1987).

14. W. J. Tropf, M. E. Thomas, and T. J. Harris, in Handbook of Optics, M. Bass, E. W. Van Stryland, D. R. Williams, and W. L. Wolfe, eds. (McGraw-Hill, 1995), Vol. II, Chap. 33. 